\title{
Thermo-mechanical behaviors of thermoplastic starch derived from sugar palm tree (Arenga pinnata).
}

\begin{abstract}
In recent years, increasing environmental concerns focused greater attention on the development of biodegradable materials. A thermoplastic starch derived from bioresources, sugar palm tree was successfully developed in the presence of biodegradable glycerol as a plasticizer. Sugar palm starch (SPS) was added with $15-40 \mathrm{w} / \mathrm{w} \%$ of glycerol to prepare workable bioplastics and coded as SPS/G15, SPS/G20, SPS/G30 and SPS/G40. The samples were characterized for thermal properties, mechanical properties and moisture absorption on exposure to humidity were evaluated. Morphological studies through scanning electron microscopy (SEM) were used to explain the observed mechanical properties. Generally, the addition of glycerol decrease the transition temperature of plasticized SPS. The mechanical properties of plasticized SPS increase with the increasing of glycerol but up to $30 \mathrm{w} / \mathrm{w} \%$. Meanwhile, the water absorption of plasticized SPS decrease with increasing of glycerol.
\end{abstract}

Keyword: Sugar palm starch; Glycerol; Thermal properties and mechanical properties 